\title{
Trends in antimicrobial resistance in Shigella species in Karachi, Pakistan
}

\author{
Erum Khan, Kausar Jabeen, Muslima Ejaz, Jaweriah Siddiqui, Muhammad Farrukh Shezad, \\ Afia Zafar
}

Department of Pathology and Microbiology, Aga Khan University Hospital, Stadium Road, PO Box 3500, Karachi 74800, Sindh, Pakistan

\begin{abstract}
Background: Shigellosis is a common cause of morbidity, especially in the very young and old, in developing countries. The disease is treated with antibiotics. Surveillance of antimicrobial resistance trends is essential owing to the global emergence of antimicrobial resistance. Methodology: The study involved 1,573 isolates of Shigella species (1996-2007) that were analyzed for trends in antimicrobial resistance. Results: The majority of the specimens $(1046 ; 66.5 \%)$ were from the pediatric population, and of these $887(84.8 \%)$ were under 5 years of age $(\mathrm{p}=0.001)$. S. flexineri was the most frequent species $(54.5 \%)$ isolated. Isolation of $S$. sonnei increased from $15.4 \%(1996)$ to $39 \%$ (2007) $(\mathrm{p}=0.001)$. Although none of the isolates was found sensitive to all the antibiotics tested, 58\% $(\mathrm{n}=907)$ were resistant to ampicillin and $85 \%(n=1,338)$ were resistant to trimethoprim-sulfamethoxazole (TMP-SMX). Out of a total of $198(12.6 \%)$ nalidixic acid resistant isolates, $6(3.0 \%)$ were also resistant to ofloxacin. Overall $1.7 \%$ of isolates were resistant to ofloxacin, $2.4 \%$ to ceftriaxone and $2.3 \%$ were resistant to combination of ampicillin, nalidixic acid and TMP-SMX.

Conclusion: Ofloxacin is still an effective drug for treatment of acute shigellosis in Pakistan. Emergence of resistance to ceftriaxone in Shigella may have grave implications in treatment of severe shigellosis in very young patients.
\end{abstract}

Keywords: shigellosis, resistance, ofloxacin, ceftriaxone, Pakistan

J Infect Dev Ctries 2009; 3(10):798-802.

(Received 12 September 2009 - Accepted 12 September 2009)

Copyright () 2009 Khan et al. This is an open-access article distributed under the Creative Commons Attribution License, which permits unrestricted use, distribution, and reproduction in any medium, provided the original work is properly cited.

\section{Introduction}

Infection by any of the Shigella species, namely S. dysenteriae, S. flexneri, S. boydii and S. sonnei, may lead to shigellosis. Global burden of shigellosis is estimated to be approximately 164.7 million cases annually with approximately 163.2 million cases from developing countries [1]. Prompt treatment with appropriate antimicrobial agents not only shortens the duration and severity of the illness but also reduces microbial carriage and thus spread of infection in the community.

Emergence of antimicrobial resistance has complicated the empirical therapy for treatment of shigellosis. Ampicillin was replaced by TMP-SMX and nalidixic acid as preferred drugs owing to emergence of resistance in the early 1970s [2]. Resistance to TMP-SMX made prominence in the 1980s with reports of resistant isolates from many countries [3]. More recently, strains resistant to nalidixic acid and those with high Minimum Inhibitory Concentration (MIC) for fluoroquinolone have been reported $[3,4,5]$, alerting clinicians to probable treatment failure. Reports of ceftriaxone resistance in Shigella spp. from various countries owing to ESBL production is another major concern $[5,6]$.

Developing countries such as Pakistan lack infrastructure to monitor anti-microbial resistance at the national level; therefore, guidelines for antimicrobial treatment are limited. Over-the-counter drug availability and self-medication instigates rapid selection and emergence of resistant strains in the community.

This study was conducted to analyze the antibiotic resistance trend in the Shigella species at our center to guide clinicians regarding the treatment options of shigellosis in Pakistan. Since Pakistan is one of the most commonly reported regions of imported cases of shigellosis in the developed world, the results of our study would be of international interest. To the best of our knowledge, this is the largest dataset of Shigella isolates reported from Pakistan.

\section{Materials and methods}

Location and sampling 
Figure 1. Frequency of different species of Shigella isolates detected at clinical laboratory of Aga Khan University Hospital Karachi (1996-2007).

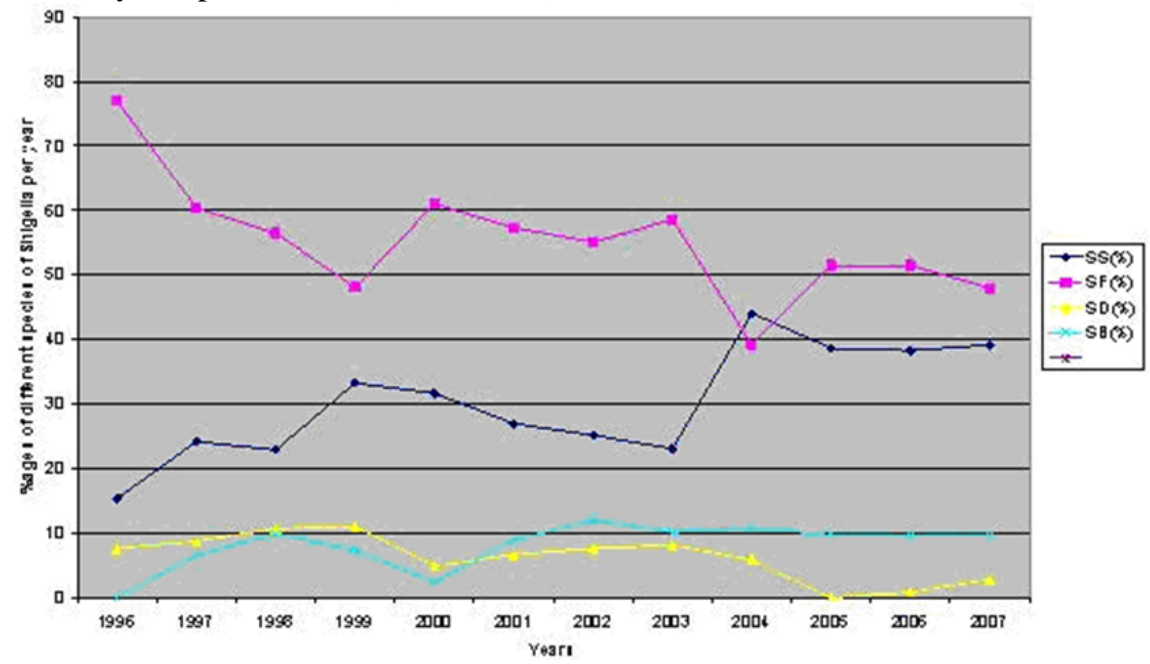

Aga Khan University Hospital (AKUH) is a 550bed tertiary care centre located in Karachi, Pakistan. The clinical microbiology laboratory receives samples across the country via its satellite units in more that 50 cities and towns. We analyzed all stool samples submitted for bacterial culture in the laboratory between the years 1996 and 2007. Stool samples were processed according to the standard methodology. Colonies morphologically suggestive of Shigella species were identified by conventional biochemical reactions (urea, citrate, triple sugar iron, indole, motility) and slide agglutination tests using antisera from Denka Seiken Co. Ltd, Japan. Nonserotypable isolates were further checked by API 20 E (Bio Murex, France). Antibiotics susceptibility was performed by Kirby Bauer disk diffusion method, against Ampicillin (10ug), Ceftriaxone (30ug), Cotrimoxazole (TMP-SMX) (25ug), Nalidixic acid (30ug) and Ofloxacin (5ug). MIC of selected agents was performed by E-Test (AB Biodisk, Solna, Sweden) according to the manufacturer's recommendations. ESBL production was detected by combined disc method (ceftriaxone alone and ceftriaxone-clavulanic acid). Escherichia coli ATCC 25922 and Klebsiella pneumoniae ATCC 700603 were used as the quality control strains.

\section{Definitions}

The pediatric population was defined as children younger than 15 years of age. The antibiotic resistance pattern was defined as follows: Pan sensitive Shigella (PSS) Shigella isolates sensitive to all the antibiotics tested. The multi-drug resistant Shigella species (MDR) was defined as strain resistant to ampicillin, TMP-SMX, and nalidixic acid. MDR isolates with additional resistance to ofloxacin and /or ceftriaxone were defined as extremely resistant strains (XDR).

\section{Statistical analysis}

Analysis was performed using SPSS version 15.0 for Windows (Statistical Package for Social Sciences, Chicago IL, USA). Mean median, standard deviations (SD) and interquartile ranges (IQR) were calculated for continuous variables. Categorization of independent continuous variables and categorical variables were calculated in a statistically meaningful way.

Descriptive analysis of age, gender, location and other characteristics were computed separately for our study population. The mean and standard deviation was computed for continuous variable by independent t-test. Proportions for categorical variables were calculated and compared by chi-square test.

A detailed stratum specific analysis on ceftriaxone resistance was done among different age groups, different Shigella species, and patients' status.

\section{Results}

Age and demographics

During the study period (1996-2007) a total of 1,573 samples were positive for Shigella species. The majority $(1046 ; 66.5 \%)$ were from the pediatric population, and of these $887(84.8 \%)$ were under 5 years of age $(p=0.001)$. 
Table 1. Percent resistance of Shigella species over period of twelve years isolated at Clinical Laboratory of Aga Khan University Hospital Karachi (1996-2007).

\begin{tabular}{|l|ccccccccccccc|c|}
\hline & $\mathbf{1 9 9 6}$ & $\mathbf{1 9 9 7}$ & $\mathbf{1 9 9 8}$ & $\mathbf{1 9 9 9}$ & $\mathbf{2 0 0 0}$ & $\mathbf{2 0 0 1}$ & $\mathbf{2 0 0 2}$ & $\mathbf{2 0 0 3}$ & $\mathbf{2 0 0 4}$ & $\mathbf{2 0 0 5}$ & $\mathbf{2 0 0 6}$ & $\mathbf{2 0 0 7}$ & Total & \multirow{2}{*}{ P- } \\
\cline { 2 - 7 } & $\mathbf{n = 2 6}$ & $\mathbf{n = 9 1}$ & $\mathbf{n = 1 3 1}$ & $\mathbf{n = 5 4}$ & $\mathbf{n = 4 1}$ & $\mathbf{n = 8 9}$ & $\mathbf{n = 2 7 4}$ & $\mathbf{n = 3 5 5}$ & $\mathbf{n = 1 0 2}$ & $\mathbf{n = 1 9 4}$ & $\mathbf{n = 1 1 5}$ & $\mathbf{n = 1 0 1}$ & $\mathbf{n = 1 5 7 3}$ & value \\
\hline SXT & 69.2 & 78 & 90.1 & 83.3 & 80.5 & 84.3 & 83.2 & 92.1 & 83.3 & 87.1 & 73.9 & 83.2 & 85.1 & $<0.001$ \\
AMP & 73.1 & 63.7 & 57.3 & 59.3 & 58.5 & 51.7 & 60.6 & 64.5 & 37.3 & 58.8 & 51.3 & 46.5 & 57.7 & 0.002 \\
C & 0 & 0 & 2.3 & 11.1 & 29.3 & 33.7 & 34.3 & 47.3 & 27.5 & 37.1 & 37.4 & 37.6 & 31.4 & $<0.001$ \\
NA & 0 & 1.1 & 6.1 & 7.4 & 7.3 & 2.2 & 4.7 & 9.9 & 19.6 & 23.0 & 22.6 & 41.6 & 12.6 & $<0.001$ \\
OFX & 0 & 0 & 0 & 0 & 0 & 1.1 & 0.4 & 1.1 & 2 & 1 & 4.3 & 10.9 & 1.7 & $<0.001$ \\
CRO & 0 & 0 & 0 & 0 & 0 & 2.2 & 1.1 & 0.8 & 4 & 8.2 & 2 & 8 & 2.4 & $<0.001$ \\
PSS & 0 & 0 & 0 & 0 & 0 & 0 & 0 & 0 & 0 & 0 & 0 & 0 & 0 & - \\
MDR & 0 & 1 & 4 & 7 & 2 & 1 & 3 & 1 & 1 & 3 & 1 & 5 & 2.3 & \\
XDR & 0 & 0 & 0 & 0 & 0 & 0 & 0 & 0 & 0 & 0 & 0 & 0 & 0 & - \\
\hline
\end{tabular}

AB: Antibiotic; SXT: septran; AMP: ampicillin; C: chloramphenicol; NA: nalidixic acid; OFX: ofloxacin; CRO Ceftriaxone, PSS: Pan Sensitive Shigella; MDR: resistant to three first-line drugs; XDR: MDR + resistance to ofloxacin and/ or ceftriaxone

\section{Monthly distribution}

Month-wise distribution of Shigella isolation was analyzed during the study period. Shigella isolation occurred throughout the year, with significant increases noted in July and August, corresponding with the monsoon season.

\section{Microbiology}

S. flexneri was the most frequent species (54.5\%) identified in both the adult and pediatric age groups. Isolation of $S$. sonnei progressively increased from $15.4 \%(\mathrm{n}=4)$ in 1996 to $33.3 \%(\mathrm{n}=18)$ in 1999 and peaked in 2004 to $44.1 \%(\mathrm{n}=45 ; \mathrm{p}=0.001)$. Peak isolation of $S$. sonnei corresponded with a relative fall of S. flexneri in that period (Fig.1). Percent isolation of $S$. boydii isolates increased from $0 \%$ in 1996 to a peak of $10.1 \%$ in $2003(\mathrm{n}=36)$. S. dysentriae isolation decreased from $7.7 \%$ in 1996 to $0 \%$ in 2005 $(\mathrm{p}=0.001)$.

\section{Antibiotic resistance}

During the study period resistance to ampicillin and cotrimoxazole remained high with fluctuations. Significant increases in resistance were noted for chloramphenicol, nalidixic acid, and ofloxacin. Ceftriaxone resistance emerged in 2001 and increased to $8 \%$ in year 2007. None of the isolates was found to be PSS i.e. sensitive to all the antibiotics tested. A large proportion of isolates was resistant to ampicillin i.e.907 (58\%) and TMP-SMX i.e.1338 (85\%); 797 $(50.7 \%)$ of isolates had co-resistance to both drugs (Table 1).

Ampicillin resistance was noticed in all four species, the highest being in S. flexneri ( $\mathrm{n}=763$; $84.1 \%)$, whereas $4.4 \%(\mathrm{n}=40) S$. sonnei isolates were resistant to ampicillin. Resistance to TMP-SMX was more common among $S$. flexneri isolates than that of $S$. sonnei $(54.6 \%$ vs. $33.0 ; \mathrm{p}=0.001)$.

Resistance to chloramphenicol emerged in 1998 $(2.3 \% ; \mathrm{n}=3)$, and increased to $37.6 \%(\mathrm{n}=38)$ in $2007(\mathrm{p}=0.001)$. Chloramphenicol resistance was more frequent in S. flexneri $(86.2 \%)$ followed by $S$. dysentriae and $S$.boydii $(6.3 \%$ and $4.7 \%$ respectively).

Shigella isolates resistant to nalidixic acid were first noted in 1997 with the highest number of resistant isolates $44(23 \%)$ in 2005. Overall resistance to nalidixic acid was common in $S$. sonnei $(\mathrm{n}=116$; $28.5 \%)$ and rare in S. flexneri $(\mathrm{n}=25 ; 3.2 \% ; \mathrm{p}=$ $0.001)$. Resistance to ofloxacin emerged in 2001 and increased to $10.9 \%$ in $2007(n=11 ; p=0.001$.

The most alarming finding of this study was detection of resistance to ceftriaxone that emerged in 2001: $65.8 \%(n=25)$ of ceftriaxone resistant isolates were from patients between two and five years of age. Resistance was mainly seen in S. flexneri $(\mathrm{n}=27$; $71.1 \%)$ followed by $S$. sonnei $(\mathrm{n}=10 ; 26.3 \%)$. The highest number of ceftriaxone resistant isolates was detected in 2005, $\mathrm{n}=16(8.2 \%)$. Prior to 2005 ceftriaxone resistance was tested by disc diffusion method; however, from 2005 onwards (due to heightened alert) all resistant isolates were reconfirmed by MIC using E-test (MIC values > $32 \mathrm{mg} / \mathrm{dl}$ ). Ceftriaxone resistant isolates were also resistant to other first line drugs; however, none of these isolates was found resistant to ofloxacin. All the ceftriaxone resistant Shigella spp. were ESBL producers.

Thirty-six $(2.3 \%)$ of the total isolates were resistant to a combination of ampicillin, nalidixic acid and TMP-SMX (MDR). None of the MDR isolates showed additional resistance to ofloxacin and /or ceftriaxone $(\mathrm{XDR}=0)$. 


\section{Discussion}

Shigellosis is primarily a disease of children and young adults. The majority of shigellosis cases in our population was seen in patients younger than five years. Transmission of Shigella commonly occurs by person-to-person contact and through food, water and fomites [7]. Flies play important role in the spread of Shigella [8]; therefore, in endemic countries, shigellosis occurs throughout the year peaking during the hot humid and rainy season [9]. Results of our study also reflect this finding with an increase of cases noted in monsoons.

S. flexneri is reported as the most frequent species from Asia [5,10,11]. Although our findings are consistent with this data, our study has revealed an epidemiological shift with progressive increases in $S$. sonnei and $S$. boydii during the study period. Changing epidemiology of shigellosis has been reported from other countries. S. flexneri was the most frequent serotype in the United States of America in the early 1960s, but that was replaced by S. sonnei during 1964-68 [12]. A recent report from Iran has indicated a shift from $S$. flexneri to $S$. sonnei as the most frequently isolated serotype [13]. Compared with previous reports, Lorenz et al. has reported increase in number of $S$. boydii in Bangladesh [11]. Follow-up studies are required to observe the persistence of this finding in our setup.

Antibiotic resistance among Shigella species is increasingly being reported from developed as well as developing countries [4,5]. None of the isolates over the period of twelve years in our study was pan sensitive. The proportion of isolates resistant to ampicillin, TMP-SMX, and both increased substantially over the study period. These agents are therefore no longer appropriate for the empirical treatment in our population.

The role of nalidixic acid as an empirical drug for treatment of acute shigellosis in young children is also being compromised in our population, as $12.6 \%$ of Shigella isolates were resistant to nalidixic acid and of these $3.8 \%$ were also resistant to ofloxacin. Our findings are consistent with data from other developing as well as developed countries $[3,4,5]$. In addition, nalidixic acid resistant isolates with high MIC for higher generation quinolones have also been reported from the United Kingdom [3].

Overall resistance to ofloxacin in our study isolates was low $(1.7 \%)$, suggesting that fluoroquinolone is still an effective drug for treatment of acute shigellosis in our population; however, treatment failures due to resistant strains can occur. It is therefore important that Shigella isolates be tested for resistance before treatment is commenced by nalidixic acid and higher generation fluoroquinolones.

Resistance to ceftriaxone has been previously reported from Asian countries [10]. A single case report of ceftriaxone resistant $S$. flexneri was reported from our center in early 2005 [14]; subsequently, the clinical laboratory was put on high alert and all isolates detected (on disc diffusion method) thereafter were confirmed by MICs. Third-generation cephalosporins are mainstays in the treatment of severe forms of diarrhoeal infections, especially in very young children in whom the use of fluoroquinolone is restricted. Resistance to this group of antibiotics is alarming, especially in the backdrop of plasmid mediated extended spectrum betalactamase (ESBL) producing Klebsiella and other Enterobacteriaceae which are increasingly being isolated at our center. All ceftriaxone resistant isolates were ESBL producers and further studies to identify the type of ESBL in these strains are underway at our center.

Our study has some limitations. First, it is a retrospective analysis of laboratory data and findings cannot be generalized to the entire population. The results of our study may be an overestimation of resistance among Shigella isolates due to sampling bias. Secondly, most of the antibiotic resistance was detected by disc diffusion method and MIC breakpoints for nalidixic acid and ofloxacin could not be determined. Despite these limitations, the findings of our study have identified changing epidemiology and emerging resistance in our Shigella isolates. These findings will certainly guide clinicians (local and international) to the options of treatment of shigellosis in patients within or those traveling from Pakistan.

In conclusion, our study has revealed an epidemiological shift with progressive increase in $S$. sonnei and $S$. boydii in our population. Antibiotic resistance in Shigella species at our center has increased at an alarming level. Substantial increase in ampicillin and TMP-SMX resistance precludes use of these agents for empirical treatment in our population. Fluoroquinolone is an option; however, it is important that Shigella isolates be tested for resistance before treatment is commenced by nalidixic acid and higher fluoroquinolones. Emergence of resistance to ceftriaxone in Shigella strains is a call for concern and an alarm for health 
care agencies to implement policies for prudent use of antibiotics.

\section{Acknowledgements}

This work was supported in part through a grant from the Joint Pakistan-US Academic and Research Program Higher Education Commission / Ministry of Science and Technology, Islamabad, Pakistan and USAID Islamabad, Pakistan.

\section{References}

1. Kotloff KL, Winickoff JP, Ivanoff B, Clemens JD, Swerdlow DL, Sansonetti PJ, Adak GK, Levine MM (1999) Global burden of Shigella infections: implications for vaccine development and implementation of control strategies. Bull World Health Organ 77: 651-666.

2. Bennish ML, Salam MA (1992) Rethinking options for the treatment of shigellosis. J Antimicrob Chemother 30: 243247.

3. Cheasty T, Day M, Threlfall EJ (2004) Increasing incidence of resistance to nalidixic acid in shigellas from humans in England and Wales: implications for therapy. Clin Microbiol Infect 10: 1033-1035.

4. Sivapalasingam S, Nelson JM, Joyce K, Hoekstra M, Angulo FJ, Mintz ED (2006) High prevalence of antimicrobial resistance among Shigella isolates in the United States tested by the national antimicrobial resistance monitoring system from 1999 to 2002. Antimicrob Agents Chemother 50: 4954.

5. Rahman M, Shoma S, Rashid H, El Arifeen S, Baqui AH, Siddique AK, Nair GB, Sack DA (2007) Increasing spectrum in antimicrobial resistance of Shigella isolates in Bangladesh: resistance to azithromycin and ceftriaxone and decreased susceptibility to ciprofloxacin. J Health Popul Nutr 25: 158-67.

6. Vasilev V, Japheth R, Yishai R, Andorn N, Valinsky L, Navon-Venezia S, Chmelnitsky I, Carmeli Y, Cohen D (2007) Extended-spectrum beta-lactamase-producing Shigella strains in Israel, 2000-2004. Eur J Clin Microbiol Infect Dis 26: 189-94.

7. Islam MS, Hossain MA, Khan SI, Khan MN, Sack RB, Albert MJ, Huq A, Colwell RR (2001) Survival of shigella dysenteriae type 1 on fomites. J Health Popul Nutr 19: 177182.

8. Levine OS, Levine MM (1991) Houseflies (Musca domestica) as mechanical vectors of shigellosis. Rev Infect Dis 13: 688-696.
9. Agtini MD, Soeharno R, Lesmana M, Punjabi NH, Simanjuntak C, Wangsasaputra F, Nurdin D, Pulungsih SP, Rofiq A, Santoso H, Pujarwoto H, Sjahrurachman A, Sudarmono P, von Seidlein L, Deen JL, Ali M, Lee H, Kim DR, Han O, Park JK, Suwandono J, Ingerani, Oyofo BA, Campbell JR, Beecham HJ, Corwin AL, Clemens JD (2005) The burden of diarrhoea, shigellosis, and cholera in North Jakarta, Indonesia: Findings from 24 months surveillance. BMC Infect Dis 5: 89.

10. Kuo CY, Su LH, Perera J, Carlos C, Tan BH, Kumarasinghe $\mathrm{G}$, So T, Van PH, Chongthaleong A, Song JH, Chiu $\mathrm{CH}$ (2008) Antimicrobial susceptibility of Shigella isolates in eight Asian countries, 2001-2004. J Microbiol Immunol Infect 41: 107-11.

11. Von Seidlein L, Kim DR, Ali M, Lee H, Wang XY, Theim VD, Canh DG, Chaicumpa W, Agtini MD, Hossain A, Bhutta ZA, Mason C, Sethabutr O, Talukder K, Nair GB, Deen JL, Kotloff K, Clemens J (2006) A multicentre study of Shigella diarrhoea in six Asian countries: disease burden, clinical manifestations, and microbiology. PLoS Med 3: e353.

12. Reller LB, Gangarosa EJ, Brachman PS (1970) Shigellosis in the United States: five-year review of nationwide surveillance, 1964-1968. Am J Epidemiol 91: 161-169.

13. Farshad S, Sheikhi R, Japoni A, Basiri E, Alborzi A (2006) Characterization of Shigella strains in Iran by plasmid profile analysis and PCR amplification of ipa genes. J Clin Microbiol 44: 2879-2883.

14. Sabir N, Zafar A (2005) Cephalosporin resistant Shigella flexneri from a clinical isolate $-\mathrm{a}$ rare finding. J Pak Med Assoc 55: 560-561.

\section{Corresponding Author}

Dr Erum Khan

Assistant Professor

Department of Pathology Microbiology

The Aga Khan University Hospital

Stadium Road, P.O. Box 3500, Karachi 74800

Pakistan

Tel \#: (9221) 4864530

Fax: (9221) 493 4294, 4932095

Email: erum.khan@aku.edu

Conflict of Interest: None is declared. 